\title{
FINDING KEY FACTORS AFFECTING THE LOCATIONS OF ELECTRIC VEHICLE CHARGING STATIONS: A SIMULATION AND ANOVA APPROACH
}

\author{
Tang, M..$^{* * *} ;$ Gong, D. ${ }^{* *, * * *, * * * * \#} ;$ Liu, S. ${ }^{* * * * * * *} \& \mathrm{Lu}, \mathrm{X}^{* *, * * * * *}$ \\ Talent Development Research Base on Decision and Consulting of Jiangsu Province, \\ Nanjing University of Information Science and Technology, Nanjing, China \\ ** International Center for Informatics Research, Beijing Jiaotong University, Beijing, China \\ ${ }^{* * *}$ School of Economics \& Management, Tsinghua University, Beijing, China \\ ${ }^{* * * *}$ School of Economics \& Management, Beijing Jiaotong University, Beijing, China \\ E-Mail: mincong@bjtu.edu.cn, gongdq@ @em.tsinghua.edu.cn, shfliu@ bjtu.edu.cn,xclu@bjtu.edu.cn \\ ( ${ }^{\#}$ Corresponding author)
}

\begin{abstract}
In this study, we aim to find the key factors affecting the location of electric vehicle charging stations. We first developed a Non-deterministic Polynomial (NP) model that aims to minimize the total travel distance of cars. Second, we applied an agent-based simulation algorithm to determine the optimized location for charging stations. Finally, we conducted multi-simulation and statistical analysis of passenger priority, car mileage, electric vehicle distribution and passenger distribution using a one-way analysis of variance (ANOVA). The results of this study show that priority is not a factor affecting the location of electric vehicle (EV) charging stations and that mileage, the EV distribution and the passenger distribution are factors affecting the location of EV charging stations, with exogenous variables such as the type of circuit and the voltage drawn as constants. The proposed model can help provide a reference for the location of charging stations in urban areas.

(Received, processed and accepted by the Chinese Representative Office.)
\end{abstract}

Key Words: $\quad$ Electric Vehicle, Location, Key Factors, Simulation, ANOVA

\section{INTRODUCTION}

The electric vehicle (EV) industry has developed very quickly worldwide over the previous five years, and the advantages of the EV are becoming increasingly more prominent, given that it can provide lower pollutant emissions and a less expensive price for energy consumptions $[1,2]$. However, there are a number of factors that hinder the development of the EV industry, including mileage (battery), the charging time, and charging convenience as well as the purchasing price of EVs, the performance of the vehicle, and battery recycling, among others [3-6].

Various studies have been conducted to solve the problems above. In this study, we focus on the charging station problem. As is well known, the charging time depends not only on the type of battery but also mostly on the power of charging facilities and the battery's charging efficiency [7]. For strong EV adoption, a battery swapping scheme should lead to a higher level of service [5]. In China, the majority of charging stations must be publicly owned at the first stage of EV adoption, particularly for a public EV (PUEV; in this study, PUEVs refer to taxis). The location of EV charging stations is becoming a hot topic. In the future, measures must be taken to determine where EVs will be charged [8]. Inadequate charging infrastructure and a lack of national guidance and local targeted construction planning all restrict EV development.

A number of studies have been conducted to examine the location problem of charging stations. One aim of the model is to improve the accessibility of charging, locating charging facilities near the urban activity centres of EV owners to maximize overall accessibility [5]. There are set covering or $P$-median facility location models [9]; the maximize flow is captured 
subject to budget constraints: the flow capturing facility location model (FCLM) [10, 11]; there are models that minimize costs while enforcing a recharging logic to ensure that all flows are served $[12,13]$; hybrid models consider both point and O-D demands [14, 15].

The factors related to the location of charging stations are very complex due to their peculiarities, such as the type of circuit, the voltage drawn, the load added to the circuit, and the charging time [16] as well as the numbers of EVs [17]. Existing studies have defined these characteristics in similar ways. In Axsen and Kurani's work [18], surveys and driving diaries are used to collect consumer information regarding consumers' willingness to buy PHEVs and what types of PHEVs they will buy. We should minimize costs while enforcing a recharging logic to ensure that all flows are served [19]. The modelling framework is applied to determine an optimal allocation of a given number of public charging stations, considering the destination and route choices, among other things [20]. What about factors related to the passenger priority, car mileage, EV distribution and passenger distribution? It is difficult for the proposed algorithms to cover most factors, which may be decisive or redundant for solving the problem; if the factors are not reasonable or redundant, they can damage the reliability of the model results.

In this study, we attempt to find the key factors affecting the location of EV charging stations. Although the model may not be entirely realistic, it provides a good idea of what impacts EV charging station locations in a basic circuit. We organize this study as follows: first, we present the model, which provides the foundation of the research problem. Then, we present the simulation algorithm with AnyLogic. In section 4, we present the simulation results through which we verify the model. Finally, we conclude this study.

\section{THE MODEL}

It has been assumed that EV adoption will occur slowly and that utilities will have enough time to adjust the current networks [5]. We assume that all EVs can directly obtain fast charging and that they do not need to wait to charge. All EVs move in a linear route.

Considering $g_{i c}$ and $\lambda$, the minimum moving distance of EVs is:

$$
\begin{array}{ll}
\operatorname{Min} \lambda\left(\sum_{c=1}^{s} \sum_{i=1}^{n} D_{i c}+\sum_{j=1}^{m} \sum_{c=1}^{s} D_{c j}\right) X_{i j k}, & \text { if } g_{i c}=1 \\
\operatorname{Min} \lambda\left(\sum_{c=1}^{s} \sum_{i=1}^{n} D_{i c}+\sum_{j=1}^{m} \sum_{i=1}^{n} L_{i j}\right) X_{i j k}, & \text { if } g_{i c}=0
\end{array}
$$

where: $\lambda$ is the coefficient of the route, $X_{i j k}$ is passenger $j$ served by vehicle $i$ at sequence $k, g_{i c} i$ s vehicle $i$ charging at station $m, s$ is the number of charging stations, $n$ is the number of vehicles, $m$ is the demand quantity, $D_{i c}$ is the distance between car $i$ and charging station $c, D_{c j}$ is the distance between passenger $j$ and charging station $c$, and $L_{i j}$ is the distance between car $i$ and passenger $j$.

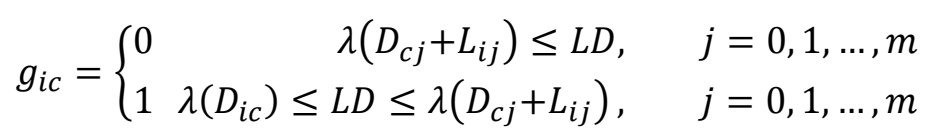

where: $L D$ is the remaining mileage.

If $L D$ is more than the sum of the distance between the demand point and the EV and the distance between the demand point and charging station, then the car will go to the demand point. If $L D$ is less than the sum of the distance between the demand point and the EV and the distance between the demand point and the charging station, while $L D$ is also more than the distance between the EV and the charging station's coordinates, then the car will go for fast charging. 
Subject to:

$$
\begin{gathered}
\sum_{i=0}^{n} \sum_{j=0}^{m} X_{i j k}=1, \quad i=0,1, \ldots, n \\
\sum_{j=0}^{m} X_{i j k} \leq 1, \quad j=0,1, \ldots, m \\
\operatorname{Max}\left(\sum_{j=1}^{m} \sum_{i=1}^{n} L_{i j}\right) \leq T D \\
\operatorname{Max}\left(\sum_{c=1}^{s} \sum_{i=1}^{n} D_{i c}\right)
\end{gathered}
$$

Eq. (4) indicates that the passenger demand is assigned to the specific car at one time. Only one passenger can be served by the car at a time (5); if the maximum distance between the demand point and any EV's coordinates is less than the mileage of the EV (6), then we should make full use of power (7). Considering people's preferences and service priority $P_{j}$ for moving, the multi-objective programming problem is:

$$
\begin{gathered}
\operatorname{Min} \lambda \alpha\left(\sum_{c=1}^{s} \sum_{i=1}^{n} D_{i c}+\sum_{j=1}^{m} \sum_{c=1}^{s} D_{c j}\right) X_{i j k} P_{j}-\lambda(1-\alpha)\left(\sum_{c=1}^{s} \sum_{i=1}^{n} D_{i c}\right) X_{i j k} P_{j}, \quad \text { if } g_{i c}=1 \\
\operatorname{Min} \lambda \alpha\left(\sum_{c=1}^{s} \sum_{i=1}^{n} D_{i c}+\sum_{j=1}^{m} \sum_{i=1}^{n} L_{i j}\right) X_{i j k} P_{j}, \quad \text { if } g_{i c}=0
\end{gathered}
$$

$\alpha$ is the weight, which reflects people's preference for the moving direction. $\alpha$ is given the values of $0.15,0.35,0.55,0.75$, and 0.95 . In the model, it refers to three different agents (EVs, charging stations and passengers); because the relations among agents are very complex, any change in the parameter will affect the reusability of the model. The agent-based algorithm is introduced in the next section.

\section{THE ALGORITHM DESIGN}

Once a model is developed, it becomes necessary to determine what tools will be used to complete the simulation and to gather results. The simulation simulator is the AnyLogic 6 University version; the simulation program can be compiled in Java Applets and supports working on a web page. The AnyLogic simulator is developed to build the charging station agent, the electric vehicle Agent and the destination agent.

The setting of the variables is presented in Table I. Passenger agents will randomly generate new demand and place it into the queue. EV agents will check the queue. When an EV receives a new demand, it will decide to move to either the demand point or a charging station (Fig. 1).

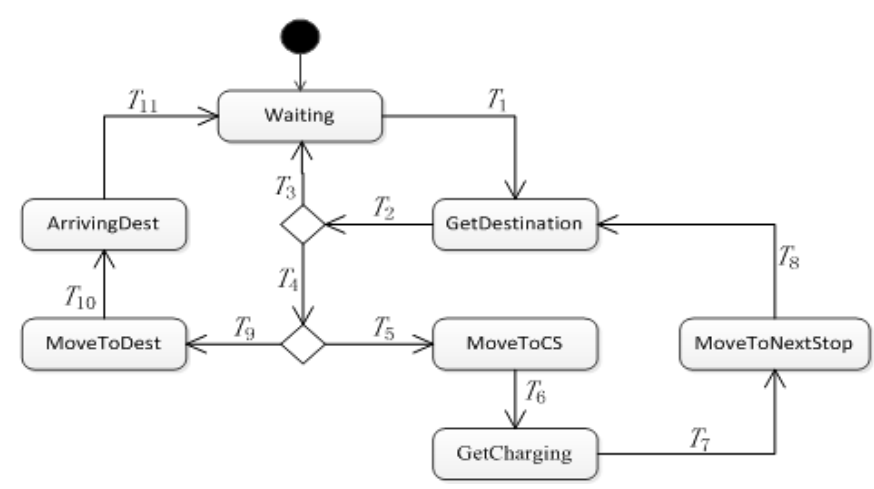

Figure 1: State diagram of agents. 
Table I: The agent settings.

\begin{tabular}{|c|c|c|}
\hline Item & Setting & Memo \\
\hline \multirow[t]{14}{*}{ Main } & (10)environment & Simulation environment \\
\hline & eCarchargestation [..] & Charging station \\
\hline & 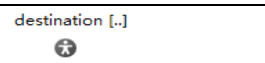 & Passenger \\
\hline & $\underset{\Theta}{e C a r[-.]}$ & $\mathrm{EV}$ \\
\hline & min $x$ & The minimum of the horizontal coordinate \\
\hline & $\max X$ & The maximum of the horizontal coordinate \\
\hline & $\min Y$ & The minimum of the vertical coordinate \\
\hline & $\max Y$ & The maximum of the vertical coordinate \\
\hline & (F) minDistance & The minimum of the distance function \\
\hline & E distance & Distance function \\
\hline & E findCar & Searching car function \\
\hline & E findClient & Searching passenger function \\
\hline & \&o requestQueue & Queuing \\
\hline & \$ event & Event \\
\hline \multirow[t]{10}{*}{ EV Agent } & (V) origin & Origin of the car \\
\hline & (1) nextNode & Different coordinates of the car \\
\hline & (v) destination & The coordinates of the passenger \\
\hline & ( $x$ & The horizontal coordinate of the car \\
\hline & y & The vertical coordinate of the car \\
\hline & (1) active & State of the car \\
\hline & (v) trip & Moving times of the car \\
\hline & travelDist & The remaining mileage \\
\hline & id & Car ID \\
\hline & (F) direct & Distance function \\
\hline \multirow[t]{6}{*}{ Passenger Agent } & (v) priority & Passenger priority \\
\hline & (1) clientId & Passenger ID \\
\hline & (v) requestingTime & The requested service time \\
\hline & (v) servicingTime & The actual service time \\
\hline & (v) & The horizontal coordinate of the passenger \\
\hline & (v) y & The vertical coordinate of the passenger \\
\hline
\end{tabular}

The output of the model includes: the requested service time (RST), the actual service time $(A S T)$, the moving distance $(R D)$, the service sequence $X_{i j k}$, and the access frequency of the charging station $(A F)$. The access frequency of the charging station is calculated when changing or considering a specific factor. Now, a one-way ANOVA is developed to find the key factors affecting the location of $\mathrm{EV}$ charging stations.

It is assumed that there are $s$ levels $\left(A_{1}, A_{2}, \ldots, A_{s}\right)$ of access frequency (AF) according to a specific factor; $n_{j}$ independent experiments are conducted in different levels $A_{j}(j=1,2, \ldots, s)$, of which the variance $\sigma^{2}$ of samples $X_{1 j}, X_{2 j}, \ldots, X_{n j}$, is the same. $X_{1 j}, X_{2 j}, \ldots, X_{n j} \sim N\left(\mu_{j}, \sigma^{2}\right)$ $(j=1,2, \ldots, s)$, and the samples are independent of each other.

$X_{i j} \sim N\left(\mu_{j}, \sigma^{2}\right), X_{i j}-\mu_{j} \sim N\left(0, \sigma^{2}\right), X_{i j}-\mu_{j}=\varepsilon_{i j}$; thus, $X_{i j}$ can be changed to: 


$$
\left\{\begin{array}{c}
X_{i j}=\mu_{j}+\varepsilon_{i j} \\
\varepsilon_{i j} \sim N\left(0, \sigma^{2}\right) \\
i=1,2, \ldots, n_{j}, j=1,2, \ldots, s
\end{array}\right.
$$

where: $\varepsilon_{i j}$ are independent from each other. Thus, we should check whether $\mu_{j}(j=1,2, \ldots, s)$ are equal to each other:

$$
\begin{aligned}
& H_{0}: \mu_{1}=\mu_{2}=\cdots \mu_{s} \\
& H_{1}: \mu_{1} \neq \mu_{2} \neq \cdots \mu_{s}
\end{aligned}
$$

Based on the hypothesis above, Eq. (10) is changed to:

$$
\left\{\begin{array}{c}
X_{i j}=\mu+\delta_{j}+\varepsilon_{i j} \\
\varepsilon_{i j} \sim N\left(0, \sigma^{2}\right) \\
i=1,2, \ldots n_{j}, j=1,2, \ldots s \\
\sum_{j=1}^{s} n_{j} \delta_{j}=0
\end{array}\right.
$$

where: $\mu=\frac{1}{n} \sum_{j=1}^{S} n_{j} \mu_{j}, \delta_{j}=\mu_{j}-\mu, j=1,2 \ldots, s$. Additionally, the hypothesis is changed to (only when $\mu_{1}=\mu_{2}=\cdots \mu_{s}, \mu_{j}=\mu, \delta_{j}=0, j=1,2, \ldots, s$ )

$$
\begin{aligned}
& H_{0}: \delta_{1}=\delta_{2}=\cdots \delta_{s}=0 \\
& H_{1}: \delta_{1} \neq \delta_{2} \neq \cdots \delta_{s} \neq 0
\end{aligned}
$$

We set:

$$
\begin{gathered}
S_{T}=\sum_{j=1}^{s} \sum_{i=1}^{n_{j}}\left[\left(X_{i j}-\overline{X_{\cdot \jmath}}\right)+\left(\overline{X_{\cdot \jmath}}-\bar{X}\right)\right]^{2} \\
=\sum_{j=1}^{s} \sum_{i=1}^{n_{j}}\left[\left(X_{i j}-\overline{X_{\cdot \jmath}}\right)^{2}+\sum_{j=1}^{s} \sum_{i=1}^{n_{j}}\left[\left(\overline{X_{\cdot \jmath}}-\bar{X}\right)^{2}+2 \sum_{j=1}^{s} \sum_{i=1}^{n_{j}}\left(X_{i j}-\overline{X_{\cdot \jmath}}\right)\left(\overline{X_{\cdot \jmath}}-\bar{X}\right)\right.\right. \\
=\sum_{j=1}^{s} \sum_{i=1}^{n_{j}}\left[\left(X_{i j}-\overline{X_{\cdot \jmath}}\right)^{2}+\sum_{j=1}^{s} \sum_{i=1}^{n_{j}}\left[\left(\overline{X_{\cdot \jmath}}-\bar{X}\right)^{2}\right.\right.
\end{gathered}
$$

where:

$$
\bar{X}=\frac{1}{n} \sum_{j=1}^{s} \sum_{i=1}^{n_{j}} X_{i j}, \overline{X_{\cdot J}}=\frac{1}{n_{j}} \sum_{i=1}^{n_{j}} X_{i j}
$$

Additionally, we set: $S_{T}=S_{E}+S_{A}$, where:

We can obtain:

$$
S_{E}=\sum_{j=1}^{s} \sum_{i=1}^{n_{j}}\left[\left(X_{i j}-\overline{X_{. J}}\right)^{2}, S_{A}=\sum_{j=1}^{s} \sum_{i=1}^{n_{j}}\left[\left(\overline{X_{\cdot}}-\bar{X}\right)^{2}\right.\right.
$$

$$
\begin{gathered}
S_{E}=\sum_{i=1}^{n_{1}}\left[\left(X_{i 1}-\overline{X_{\cdot 1}}\right)^{2}+\cdots+\sum_{i=1}^{n_{s}}\left[\left(X_{i s}-\overline{X_{\cdot s}}\right)^{2}\right.\right. \\
\sum_{i=1}^{n_{j}}\left[\frac{\left(X_{i j}-\overline{X_{\cdot}}\right)^{2}}{\sigma^{2}} \sim \chi^{2}\left(n_{j}-1\right), \frac{s_{E}}{\sigma^{2}} \sim \chi^{2}(n-s)\right. \\
S_{A}=\sum_{j=1}^{s} \sum_{i=1}^{n_{j}}\left[\left(\overline{X_{\cdot J}}-\bar{X}\right)^{2}=\sum_{j=1}^{s} n_{j} \bar{X}_{\cdot j}^{-2}-n \bar{X}^{2}\right.
\end{gathered}
$$

Thus, the degree of freedom of $S_{A}$ is:

$$
\begin{gathered}
s-1\left(\sum_{j=1}^{S} \sqrt{n_{j}}\left[\sqrt{n_{j}}\left(\overline{X_{. j}}-\bar{X}\right)\right]=\sum_{j=1}^{S} \sum_{i=1}^{n_{j}} X_{i j}-n \bar{X}=0\right) \\
E\left(S_{A}\right)=(s-1) \sigma^{2}+\sum_{j=1}^{s} n_{j} \delta_{j}^{2}
\end{gathered}
$$

Thus, $E\left(S_{A} / s-1\right)=\sigma^{2}$ when $\delta_{1}=\delta_{2}=\ldots=\delta_{s}=0$; otherwise,

$$
E\left(S_{A} / s-1\right)=\sigma^{2}+\frac{1}{s-1} \sum_{j=1}^{s} n_{j} \delta_{j}^{2}>\sigma^{2}
$$

$E\left(S_{E} / n-s\right)=\sigma^{2}$, when $\delta_{1}=\delta_{2}=\ldots=\delta_{s}=0$.

$$
\frac{s_{A} / s-1}{S_{E} / n-s}=\frac{\frac{\frac{s_{A}}{\sigma^{2}}}{s-1}}{\frac{\frac{s_{E}}{\sigma^{2}}}{n-s}} \sim F(s-1, n-s)
$$


$F=\frac{s_{A} / s-1}{S_{E} / n-s}$ becomes larger when $\delta_{j}(j=1,2, \ldots, s)$ are not equal to each other. Thus, the critical region is $F=\frac{s_{A} / s-1}{S_{E} / n-s} \geq k$, where the value of $k$ can be obtained by $F_{\alpha}(s-1, n-s)$.

If $F=\frac{s_{A} / s-1}{s_{E} / n-s} \geq F_{\alpha}(s-1, n-s)$, then $\delta_{1} \neq \delta_{2} \neq \ldots \neq \delta_{s} \neq 0, \mu_{1} \neq \mu_{2} \neq \ldots \neq \mu_{s}$; thus, the specific factor plays an important role in the location of EV charging stations.

If $F=\frac{s_{A} / s-1}{s_{E} / n-s} \leq F_{\alpha}(s-1, n-s)$, then $\delta_{1}=\delta_{2}=\ldots=\delta_{s}=0, \mu_{1}=\mu_{2}=\ldots=\mu_{s}$; thus, the specific factor cannot affect the location of EV charging stations.

\section{CASE STUDY: CITY OF BEIJING}

There are 13 charging stations, e.g., Datun station, Huixinxiqiao station, and Beitucheng station, among others, which are often used in the central area of Beijing (Fig. 2). The blue line is a practical road, and the red spot is a charging station.

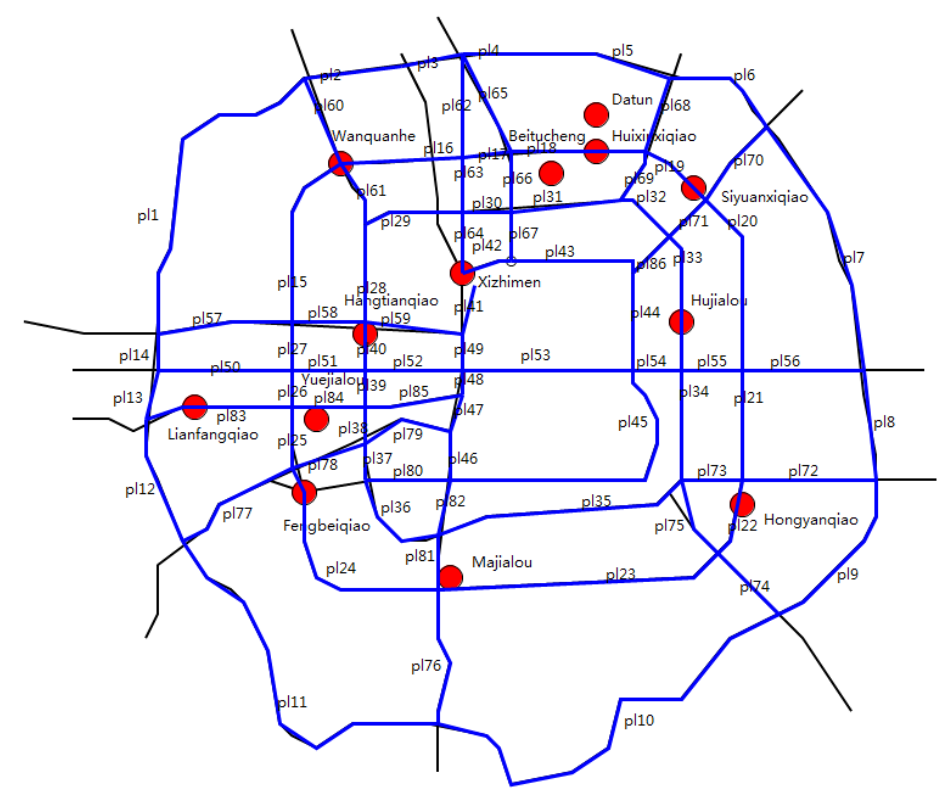

Figure 2: The location of charging stations in Beijing.

Given the location of charging stations in Beijing, the aim of this study is to optimize the existing locations. The initial parameters settings are presented in Table II.

Table II: The initial parameter settings.

\begin{tabular}{|c|l|c|}
\hline Parameter & \multicolumn{1}{|c|}{ Memo } & Distribution (value) \\
\hline$s$ & Charging station agent & 13 \\
\hline$m$ & Passenger agent & 100 \\
\hline$n$ & EV agent & 10 \\
\hline$x$ & The horizontal coordinate of the agent & $U(-410,240)$ \\
\hline$y$ & The vertical coordinate of the agent & $U(120,770)$ \\
\hline$T D$ & Vehicle range & 600 \\
\hline$\lambda$ & Coefficient of the route & 1.4 \\
\hline$P_{j}$ & Service priority & $\operatorname{Random}()$ \\
\hline
\end{tabular}

This study uses the AnyLogic simulator to analyse the access frequency of charging stations to find the factors affecting the location of charging stations. This simulator randomly generates information on 100 passengers (coordinates, priority), information on $10 \mathrm{EVs}$ (coordinates, state 
is idle), and information on 10 charging stations, as shown in Fig. 1. The results for 100 iterations are shown in Table III.

Table III: Parts of the simulation results.

\begin{tabular}{|c|c|c|c|c|c|c|c|c|c|}
\hline $\boldsymbol{O C C}(\boldsymbol{x})$ & $\boldsymbol{O C C}(\boldsymbol{y})$ & $\boldsymbol{C P}(\boldsymbol{x})$ & $\boldsymbol{C P}(\boldsymbol{y})$ & $\boldsymbol{C S I D}$ & $\boldsymbol{L D}$ & $\boldsymbol{P I D}$ & $\boldsymbol{P P}$ & $\boldsymbol{C I D}$ & $\boldsymbol{R D}$ \\
\hline 218 & 439 & -234 & 188 & 0 & 600 & 37 & 0.91 & 10 & 517 \\
\hline-223 & 224 & -221 & 286 & 0 & 600 & 36 & 0.82 & 10 & 62 \\
\hline-221 & 286 & 15 & 263 & 0 & 537 & 16 & 0.76 & 10 & 237 \\
\hline 110 & 500 & 190 & 614 & 0 & 600 & 8 & 0.72 & 10 & 139 \\
\hline-120 & 310 & -241 & 209 & 0 & 600 & 19 & 0.59 & 10 & 157 \\
\hline-130 & 560 & -37 & 677 & 0 & 600 & 30 & 0.43 & 10 & 150 \\
\hline-37 & 677 & -46 & 512 & 0 & 449 & 72 & 0.28 & 10 & 165 \\
\hline 60 & 350 & -15 & 258 & 0 & 600 & 44 & 0.26 & 10 & 118 \\
\hline-15 & 258 & -53 & 473 & 0 & 481 & 49 & 0.23 & 10 & 218 \\
\hline 60 & 350 & 120 & 207 & 0 & 600 & 91 & 0.19 & 10 & 155 \\
\hline
\end{tabular}

Note: $O C C$ : the original coordinates of the car, $C P$ : the coordinates of the passenger, CSID: charging station $I D$, $L D$ : remaining mileage, $P I D$ : passenger $I D, P P$ : passenger priority, $C I D$ : car $I D, R D$ : moving distance.

When priority is considered, the access frequency of charging stations is shown in Table IV.

Table IV: Simulation results - priority is considered.

\begin{tabular}{|c|c|c|c|c|c|c|c|}
\hline \multirow{2}{*}{$\alpha=0.15$} & $C S 5$ & \multicolumn{7}{|c|}{ Experiment } & Total \\
\cline { 2 - 8 } & $R A F(\%)$ & 11 & 4 & 5.5 & 6 & 14 & 40.5 \\
\hline \multirow{2}{*}{$\alpha=0.35$} & $C S 5$ & \multicolumn{7}{|c|}{ Experiment } & Total \\
\cline { 2 - 8 } & $R A F(\%)$ & 12 & 10 & 3 & 4 & 12.5 & 41.5 \\
\hline \multirow{2}{*}{$\alpha=0.55$} & $C S 5$ & \multicolumn{7}{|c|}{ Experiment } & Total \\
\cline { 2 - 8 } & $R A F(\%)$ & 10 & 6 & 12.5 & 7.5 & 7.5 & 43.5 \\
\hline \multirow{2}{*}{$\alpha=0.75$} & $C S 5$ & \multicolumn{7}{|c|}{ Experiment } & Total \\
\cline { 2 - 8 } & $R A F(\%)$ & 5 & 12.5 & 9 & 11 & 10 & 47.5 \\
\hline \multirow{2}{*}{$\alpha=0.95$} & $C S 5$ & \multicolumn{7}{|c|}{ Experiment } & Total \\
\cline { 2 - 8 } & $R A F(\%)$ & 10 & 10.5 & 12 & 10 & 7.5 & 50 \\
\hline
\end{tabular}

$C S$ : charging station, $A F$ : access frequency, $R A F$ : ratio of access frequency (the same below). V.

When priority is not considered, the access frequency of charging stations is shown in Table

Table V: Simulation results - priority is not considered.

\begin{tabular}{|c|c|c|c|c|c|c|c|}
\hline \multirow{2}{*}{$\alpha=0.15$} & CS5 & \multicolumn{7}{|c|}{ Experiment } & Total \\
\cline { 2 - 8 } & $R A F(\%)$ & 9 & 3 & 7 & 10.5 & 8 & 37.5 \\
\hline \multirow{2}{*}{$\alpha=0.35$} & $C S 5$ & \multicolumn{7}{|c|}{ Experiment } & Total \\
\cline { 2 - 9 } & $R A F(\%)$ & 4 & 5 & 6 & 8 & 11.5 & 34.5 \\
\hline \multirow{2}{*}{$\alpha=0.55$} & $C S 5$ & \multicolumn{7}{|c|}{ Experiment } & Total \\
\cline { 2 - 8 } & RAF (\%) & 3 & 11 & 10 & 6 & 3.5 & 33.5 \\
\hline \multirow{2}{*}{$\alpha=0.75$} & $C S 5$ & \multicolumn{7}{|c|}{ Experiment } & Total \\
\cline { 2 - 8 } & RAF (\%) & 6.5 & 10 & 5 & 7.5 & 15.5 & 44.5 \\
\hline \multirow{2}{*}{$\alpha=0.95$} & $C S 5$ & \multicolumn{7}{|c|}{ Experiment } & Total \\
\cline { 2 - 8 } & $R A F(\%)$ & 15 & 5.5 & 6 & 3 & 10 & 39.5 \\
\hline
\end{tabular}


It can be observed that $s=10, n=50, S_{A}=50.925, S_{E}=497.700, s-1=9, n-s=40$, $\overline{S_{A}}=\frac{S_{A}}{(s-1)}=5.658, \overline{S_{E}}=\frac{S_{E}}{(n-s)}=12.442, F=\frac{\overline{S_{A}}}{\overline{S_{E}}}=0.455, F_{\alpha}(s-1, n-s)=1.79$, and $F \leq F_{\alpha}(s-1$, $n-s)$; thus, priority is not a factor affecting the location of EV charging stations.

In the simulation model above, the mileage $T D$ is 600; we check whether mileage will affect the location of EV charging stations. We can obtain that $s=5, n=25, S_{A}=324.960$, $S_{E}=183.300, s-1=4, n-s=20, \overline{S_{A}}=\frac{S_{A}}{(s-1)}=81.240, \overline{S_{E}}=\frac{S_{E}}{(n-s)}=9.165, F=\frac{\overline{S_{A}}}{\overline{S_{E}}}=8.864$, $F_{\alpha}(s-1, n-s)=2.25$, and $F \geq F_{\alpha}(s-1, n-s)$. Thus, mileage is a factor affecting the location of EV charging stations (Table VI).

Table VI: Mileage and access frequency ratio.

\begin{tabular}{|c|c|c|c|c|c|c|c|}
\hline \multirow{2}{*}{$T D=400$} & $C S 5$ & \multicolumn{7}{|c|}{ Experiment } & Total \\
\cline { 2 - 8 } & $R A F(\%)$ & 1 & 4 & 5 & 6 & 2 & 3.6 \\
\hline \multirow{2}{*}{$T D=500$} & $C S 5$ & \multicolumn{7}{|c|}{ Experiment } & Total \\
\cline { 2 - 8 } & $R A F(\%)$ & 6 & 2.5 & 1 & 2.5 & 4 & 3.2 \\
\hline \multirow{7}{*}{$T D=600$} & $C S 5$ & \multicolumn{7}{|c|}{ Experiment } & Total \\
\cline { 2 - 8 } & $R A F(\%)$ & 12.5 & 10 & 7.5 & 7 & 10.5 & 9.5 \\
\hline \multirow{2}{*}{$T D=700$} & $C S 5$ & \multicolumn{7}{|c|}{ Experiment } & Total \\
\cline { 2 - 8 } & $R A F(\%)$ & 14 & 9 & 5 & 6 & 15 & 9.8 \\
\hline \multirow{2}{*}{$T D=800$} & $C S 5$ & \multicolumn{7}{|c|}{ Experiment } & Total \\
\cline { 2 - 8 } & $R A F(\%)$ & 15 & 9 & 8 & 8 & 14 & 10.8 \\
\hline
\end{tabular}

In the simulation model above, the coordinates of the EV Agent have a uniform distribution; we check whether the distribution can affect the location of EV charging stations. In this model, uniform distribution, triangular distribution, normal distribution, exponential distribution and PERT distribution are considered. We can obtain that $s=5, n=25, S_{A}=44.240, S_{E}=62.700$, $s-1=4, n-s=20, \overline{S_{A}}=\frac{s_{A}}{(s-1)}=11.060, \overline{S_{E}}=\frac{S_{E}}{(n-s)}=3.135, F=\frac{\overline{S_{A}}}{\overline{S_{E}}}=3.528, F_{\alpha}(s-1, n-s)=$ 2.25 , and $F \geq F_{\alpha}(s-1, n-s)$; thus, the distribution of EVs is a factor affecting the location of $\mathrm{EV}$ charging stations (Table VII).

Table VII: Distribution and access frequency (car).

\begin{tabular}{|l|c|c|c|c|c|c|c|}
\hline \multirow{2}{*}{ Uniform distribution } & $C S 5$ & \multicolumn{5}{|c|}{ Experiment } & Total \\
\cline { 2 - 8 } & $R A F(\%)$ & 7 & 8 & 5.5 & 7.5 & 8 & 36 \\
\hline \multirow{2}{*}{ Triangular distribution } & $C S 5$ & \multicolumn{5}{|c|}{ Experiment } & Total \\
\cline { 2 - 8 } & $R A F(\%)$ & 9 & 11 & 12 & 9 & 8 & 49 \\
\hline \multirow{2}{*}{ Normal distribution } & $C S 5$ & \multicolumn{5}{|c|}{ Experiment } & Total \\
\cline { 2 - 8 } & $R A F(\%)$ & 2 & 7 & 6 & 8 & 6 & 29 \\
\hline \multirow{2}{*}{ Exponential distribution } & $C S 5$ & \multicolumn{7}{|c|}{ Experiment } & Total \\
\cline { 2 - 8 } & $R A F(\%)$ & 6 & 10 & 9.5 & 9 & 8 & 42.5 \\
\hline \multirow{2}{*}{ PERT distribution } & $C S 5$ & \multicolumn{7}{|c|}{ Experiment } & Total \\
\cline { 2 - 8 } & $R A F(\%)$ & 6 & 10 & 7 & 10 & 6 \\
\hline
\end{tabular}

In the simulation model above, the coordinates of the passenger agent have a uniform distribution; we check whether the distribution can affect the location of EV charging stations. In this model, uniform distribution, triangular distribution, normal distribution, exponential 
distribution and PERT distribution are considered. Similarly, we can obtain that $s=5, n=25$, $S_{A}=1,328.860, S_{E}=76.600, s-1=4, n-s=20, \overline{S_{A}}=\frac{S_{A}}{(s-1)}=332.215, \overline{S_{E}}=\frac{S_{E}}{(n-s)}=3.830$, $F=\overline{\overline{S_{A}}}=86.740, F_{\alpha}(s-1, n-s)=2.25$, and $F \geq F_{\alpha}(s-1, n-s)$; thus, the passenger distribution is a factor affecting the location of EV charging stations (Table VIII).

Table VIII. Distribution and access frequency (passenger).

\begin{tabular}{|l|c|c|c|c|c|c|c|}
\hline \multirow{2}{*}{ Uniform distribution } & $C S 5$ & \multicolumn{7}{|c|}{ Experiment } & Total \\
\cline { 2 - 9 } & $R A F(\%)$ & 7 & 7 & 7.5 & 8.5 & 8 & 38 \\
\hline \multirow{2}{*}{ Triangular distribution } & $C S 5$ & \multicolumn{7}{|c|}{ Experiment } & Total \\
\cline { 2 - 9 } & $R A F(\%)$ & 13.5 & 12 & 12.5 & 15 & 14.5 & 67.5 \\
\hline \multirow{6}{*}{ Normal distribution } & $C S 5$ & \multicolumn{7}{|c|}{ Experiment } & Total \\
\cline { 2 - 9 } & $R A F(\%)$ & 29 & 23 & 21 & 20 & 27 & 120 \\
\hline \multirow{2}{*}{ Exponential distribution } & $C S 5$ & \multicolumn{7}{|c|}{ Experiment } & Total \\
\cline { 2 - 9 } & $R A F(\%)$ & 3 & 1 & 2.5 & 2 & 2 & 10.5 \\
\hline \multirow{2}{*}{ PERT distribution } & $C S 5$ & \multicolumn{7}{|c|}{ Experiment } & Total \\
\cline { 2 - 8 } & $R A F(\%)$ & 11 & 11 & 10 & 8 & 10.5 & 50.5 \\
\hline
\end{tabular}

It can be concluded that priority is not a factor affecting the location of EV charging stations and that mileage, the EV distribution and the passenger distribution are factors affecting the location of EV charging stations. Private charging piles can provide a slow charge for EV due to the peaks and valleys of electricity; such charging piles are located based on the actual demand; in contrast, for public charging stations, due to their characteristics and operations, such as mileage, the EV distribution and the passenger distribution, among others, the reasonable location of charging stations plays an important role, affecting the operation of EVs. The proposed model can help provide a reference for the location of charging stations in urban areas.

\section{OPTIMIZING THE LOCATION OF CHARGING STATIONS}

In the following section, we optimize the layout for only one situation: The mileage is 600 , priority is not considered, and the EVs and the passengers have the same distribution. We set the parameters as shown in Table IX.

Table IX: Parameter settings.

\begin{tabular}{|c|l|l|c|}
\hline & Parameter & \multicolumn{1}{|c|}{ Memo } & Distribution (value) \\
\hline \multirow{2}{*}{ Passengers } & $\nabla^{x}$ & The horizontal coordinate of the agent & $U(\min X, \max X)$ \\
\cline { 2 - 4 } & $\nabla^{y}$ & The vertical coordinate of the agent & $U(\min Y, \max Y)$ \\
\hline \multirow{2}{*}{ EVs } & $\nabla^{x}$ & The horizontal coordinate of the agent & $U(\min X, \max X)$ \\
\cline { 2 - 4 } & $\nabla^{y}$ & The vertical coordinate of the agent & $U(\min Y, \max Y)$ \\
\hline Mileage & travelDist & Mileage & 600 \\
\hline
\end{tabular}

The multi-objective programming problem is changed to:

$$
\operatorname{Min} \lambda \alpha\left(\sum_{c=1}^{s} \sum_{i=1}^{n} D_{i c}+\sum_{j=1}^{m} \sum_{c=1}^{s} D_{c j}\right) X_{i j k}-\lambda(1-\alpha)\left(\sum_{c=1}^{s} \sum_{i=1}^{n} D_{i c}\right) X_{i j k}, \quad \text { if } g_{i c}=1
$$


Subject to:

$$
\operatorname{Min} \lambda \alpha\left(\sum_{c=1}^{s} \sum_{i=1}^{n} D_{i c}+\sum_{j=1}^{m} \sum_{i=1}^{n} L_{i j}\right) X_{i j k}, \quad \text { if } g_{i c}=0
$$

$$
\begin{gathered}
\sum g_{i 1} \approx \sum g_{i 2} \approx \cdots \sum g_{i s} \\
\min (s)
\end{gathered}
$$

Optimization will end when there is no significant difference in the charging frequencies (24) while maintaining the minimum number of charging stations (25).

When $\alpha=0.15$, the total distance of the moving car is $42,005.47$, whereas the shortest distance is $23,993.5$. Theoretically, the detour distance is $18,011.97$, and the detour ratio is $42.9 \%$. The statistical results are shown in Table X.

Table X: Results when $\alpha=0.15$.

\begin{tabular}{|l|c|c|c|c|c|c|c|c|c|c|c|c|c|c|}
\hline$C S$ & 1 & 2 & 3 & 4 & 5 & 6 & 7 & 8 & 9 & 10 & 11 & 12 & 13 & Total \\
\hline$A F$ & 2 & 1 & 3 & 5 & 8 & 5 & 6 & 4 & 6 & 14 & 2 & 6 & 7 & 69 \\
\hline$R A(\%)$ & 3 & 1 & 4 & 7 & 12 & 7 & 9 & 6 & 9 & 20 & 3 & 9 & 10 & 100 \\
\hline
\end{tabular}

When $\alpha=0.35$, the total distance of the moving car is $38,000.96$, whereas the shortest distance is $22,584.73$. Theoretically, eth detour distance is $15,416.23$, and the detour ratio is $40.6 \%$. The statistical results are shown in Table XI.

Table XI: Results when $\alpha=0.35$.

\begin{tabular}{|l|c|c|c|c|c|c|c|c|c|c|c|c|c|c|}
\hline$C S$ & 1 & 2 & 3 & 4 & 5 & 6 & 7 & 8 & 9 & 10 & 11 & 12 & 13 & Total \\
\hline$A F$ & 1 & 1 & 2 & 2 & 7 & 5 & 5 & 6 & 8 & 15 & 6 & 2 & 11 & 71 \\
\hline$R A(\%)$ & 1 & 1 & 3 & 3 & 9.9 & 7 & 7 & 8 & 11 & 21 & 8 & 3 & 15 & 100 \\
\hline
\end{tabular}

When $\alpha=0.55$, the total distance of the moving car is $36,369.82$, whereas the shortest distance is $22,823.29$. Theoretically, the detour distance is $13,546.53$, and the detour ratio is $37.2 \%$. The statistical results are shown in Table XII.

Table XII: Results when $\alpha=0.55$.

\begin{tabular}{|l|c|c|c|c|c|c|c|c|c|c|c|c|c|c|}
\hline$C S$ & 1 & 2 & 3 & 4 & 5 & 6 & 7 & 8 & 9 & 10 & 11 & 12 & 13 & Total \\
\hline$A F$ & 0 & 5 & 4 & 3 & 3 & 7 & 5 & 6 & 8 & 14 & 4 & 2 & 5 & 66 \\
\hline$R A(\%)$ & 0 & 8 & 6 & 5 & 4.5 & 11 & 8 & 9 & 12 & 21 & 6 & 3 & 8 & 100 \\
\hline
\end{tabular}

When $\alpha=0.75$, the total distance of the moving car is $38,331.24$, whereas the shortest distance is $22,187.44$. Theoretically, the detour distance is $16,143.8$, and the detour ratio is $42.1 \%$. The statistical results are shown in Table XIII.

Table XIII: Results when $\alpha=0.75$.

\begin{tabular}{|l|c|c|c|c|c|c|c|c|c|c|c|c|c|c|}
\hline$C S$ & 1 & 2 & 3 & 4 & 5 & 6 & 7 & 8 & 9 & 10 & 11 & 12 & 13 & Total \\
\hline$A F$ & 2 & 0 & 1 & 7 & 5 & 5 & 4 & 3 & 8 & 16 & 2 & 6 & 11 & 70 \\
\hline$R A(\%)$ & 3 & 0 & 1 & 10 & 7 & 7 & 6 & 4 & 11 & 23 & 3 & 9 & 16 & 100 \\
\hline
\end{tabular}

When $\alpha=0.95$, the total distance of the moving car is $40,085.88$, whereas the shortest distance is $22,809.36$. Theoretically, the detour distance is $17,276.52$, and the detour ratio is $43.1 \%$. The statistical results are shown in Table XIV. 
Table XIV: Results when $\alpha=0.95$.

\begin{tabular}{|l|c|c|c|c|c|c|c|c|c|c|c|c|c|c|}
\hline$C S$ & 1 & 2 & 3 & 4 & 5 & 6 & 7 & 8 & 9 & 10 & 11 & 12 & 13 & Total \\
\hline$A F$ & 1 & 1 & 2 & 8 & 6 & 5 & 4 & 3 & 7 & 14 & 3 & 13 & 5 & 72 \\
\hline$R A(\%)$ & 1 & 1 & 3 & 11 & 8 & 7 & 6 & 4 & 10 & 19 & 4 & 18 & 7 & 100 \\
\hline
\end{tabular}

It can be concluded that regardless of the value, the access frequency of charging stations 4 , $5,6,9,10,12$, and 13 is relatively high; in particular, the access frequency of charging station 10 can reach $20 \%$. In contrast, charging stations $1,2,3,7,8$, and 11 have a low access frequency; in particular, the access frequency of charging stations 1, 2 and 3 is less than $5 \%$. Thus, there are few charging stations in some areas, and charging pressure arises; although the layout density of charging stations in some areas is high, the charging stations are in an idle state all of the time. Therefore, the existing layout of charging stations in Beijing is unreasonable. There is small need to charge in the areas of charging stations 4, 5, 6, 9, 10, 12, and 13, whereas there is a large need to charge in the areas of charging stations $1,2,3,7,8$, and 11. The optimization methods used in this study include: adding options at the edge point and diluting the access frequency, among others; the aim of optimizing is to ensure that there are no large differences in the access frequency of all charging stations.

(1) Adding options at the edge point

According to the layout of Lianfangqiao station, Yuanjialou station, Feng beibridge station and Majialou station, this study selects point $A(-400,420)$, point $\mathrm{B}(-400,730)$ and point $\mathrm{C}$ $(-130,730)$ to calculate the centre of gravity, which is the added charging station. A genetic algorithm and Monte Carlo simulations can obtain the coordinates $(-265,575)$ of a new charging station (ID 14). The access frequency of charging station 5, 10, 12, 13, and 14 is relatively high (more than $10 \%$ ), whereas the access frequency of charging stations $1,2,3,4,8$, 9 , and 11 is relatively low (less than $5 \%$ ). Thus, we still need to optimize the layout of the charging stations.

(2) Diluting the access frequency

According to the layout of Majialou station $(-130,560)$, Hujialouqiao station $(60,350)$ and Hongyanqiao station $(110,350)$, the study selects point A $(-130,560)$, point $B(60,350)$ and point $C(110,350)$ to calculate the centre of gravity, which is the added charging station. A genetic algorithm and Monte Carlo simulations can obtain the coordinates $(-27,462)$ of a new charging station (ID 15). The access frequency of charging station 5, 14 and 15 is relatively high (more than $10 \%$ ), whereas the access frequency of charging stations 1,2 and 9 is relatively low (less than $5 \%$ ). Thus, we still need to optimize the layout of the charging stations.

(3) Obtaining the three vertices of the largest triangle

According to the high access frequency of the charging station layout (Xizhimenqiao station, Fengbeiqiao station and the station with ID 15), Heron's formula is developed to obtain the three vertices of the largest triangle and to calculate the center of gravity. Monte Carlo simulations give the coordinates $(-144,429)$ of a new charging station (ID 16). The access frequency of charging stations 13 and 15 is relatively high (more than $10 \%$ ), whereas the access frequency of charging stations 1, 2, 3, 7 and 11 is relatively low (less than $5 \%$ ). Thus, we still need to optimize the layout of the charging stations.

(4) Calculating the shortest distance with reference points

In this study, we choose Majialou station $(-130,560)$, Hongyanqiao station $(110,500)$, point D $(-130,730)$ and point $E(110,730)$ as the reference points to find a new point, the shortest distance to each reference point. Thus, the coordinates $(-28,632)$ of a new charging station (ID 17) are obtained. The access frequency of charging stations 13 and 15 is still relatively high (more than $10 \%$ ), whereas the access frequency of the other charging stations is maintained at $3 \%$ to $6 \%$. The charging station layout is improved to some extent, but we still need to optimize 
the layout of the charging stations. Similarly, we choose Hongyanqiao station $(110,500)$, the station with ID $17(-28,632)$, point $F(-28,730)$, point $\mathrm{G}(230,730)$ and point $\mathrm{H}(230,500)$ as the reference points. Thus, the coordinates $(94,605)$ of a new charging station (ID 18) are obtained. The access frequency of charging station 15 is still relatively high (more than $10 \%$ ), whereas the access frequency of the other charging stations is maintained at $3 \%$ to $7 \%$. The charging station layout is much improved, but we still need to optimize the layout of the charging stations.

(5) Deleting unreasonable points

According to the analysis above, we found that the access frequency of Huixinxiqiao station is very low. Thus, we attempt to find the optimized layout when the charging of Huixinxiqiao station is removed.

Finally, the access frequency of the charging stations will remain at a ratio of $4 \%$ to $7 \%$. Therefore, compared to the other optimizations, the access frequency of the charging stations is more even, and the charging station layout tends to be more reasonable. The optimized layout of EV charging stations is shown in Fig. 3.

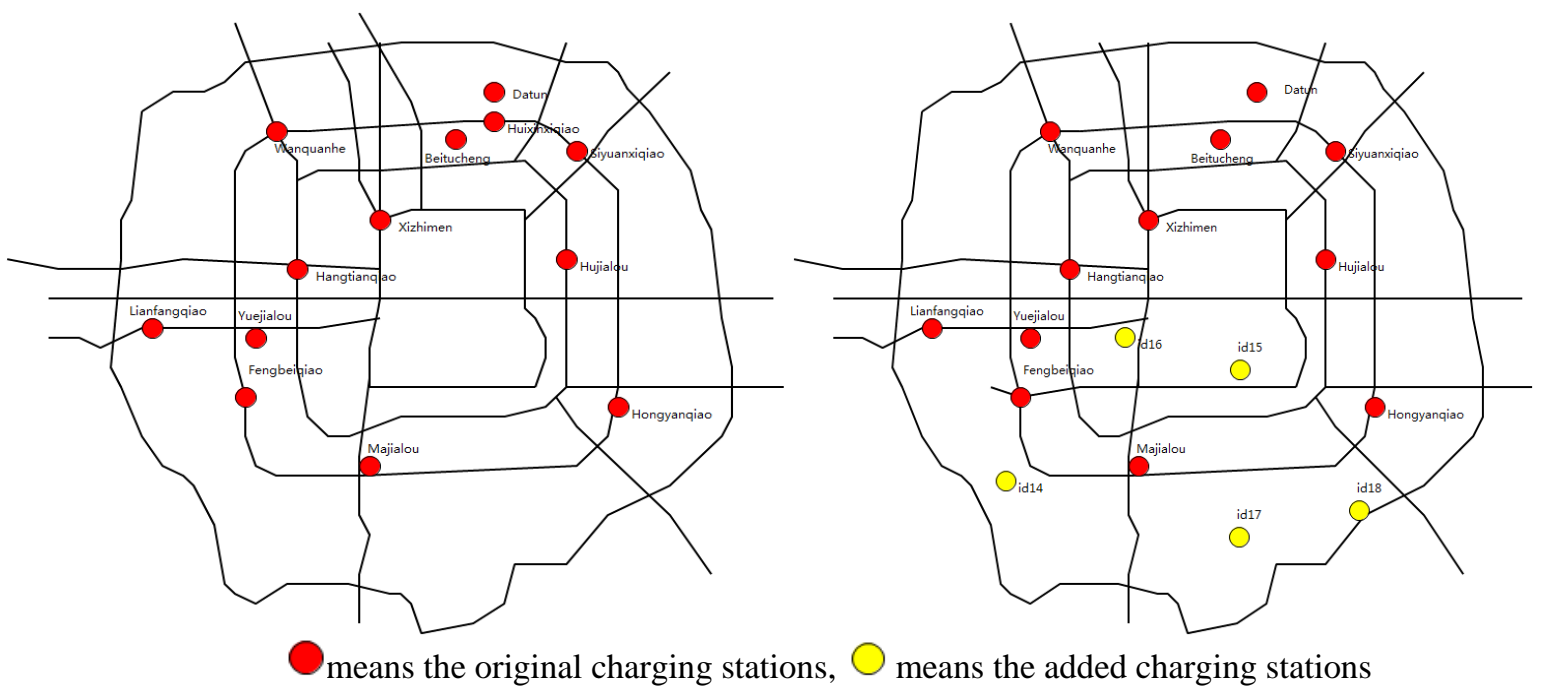

Figure 3: The optimizing layout of charging stations.

Eventually, we can obtain the coordinates of all charging stations: Datun station $(-10,182)$, Beitucheng station (-47, 228), Wanquanhe station (-223, 224), Xizhimen station $(-120,310)$, Hangtianqiao station (-200, 360), Yuejialou station (-240, 430), Lianfangqiao station $(-340,420)$, Fengbeiqiao station $(-250,490)$, Majialou station $(-130,560)$, Siyuanxiqiao station $(70,240)$, Hujialou station $(60,350)$, Hongyanqiao station $(110,500)$, the station with ID 14 $(-265,575)$, the station with ID $15(-27,462)$, the station with ID $16(-144,429)$, the station with ID $17(-28,632)$,and the station with ID $18(94,605)$.

\section{CONCLUSION}

EVs are very promising for a low carbon economy because they use electricity and produce zero pollutants. However, EV development also faces some problems. Among the problems, charging station location is one of the keys. It is found that the location of a charging station typically incorporates the type of circuit, the voltage drawn, the load on the circuit, and the amount of charging time, all of which have been well studied. We take into consideration other factors including the passenger priority, mileage, EV distribution and passenger distribution in this study and propose an optimization model. Through simulation and an ANOVA approach, we test the impacts of these specific factors on the location of EV charging stations. We find 
that priority is not a factor affecting the location of EV charging stations and that mileage, the $\mathrm{EV}$ distribution and the passenger distribution influence the location of EV charging stations. However, this study has certain limitations and deficiencies. We will consider more factors related to the location of charging stations, such as the traffic flow, practical routine, and user characteristics.

\section{ACKNOWLEDGEMENTS}

The study is supported by funding from the Beijing Natural Science Foundation (041501108), the China Postdoctoral Science Foundation (2016M591194), the Beijing Municipal Commission of Economy and Information Technology (B16M00140, B17I00110) and the National Natural Science Foundation (71132008, 71390334). We greatly appreciate their support.

\section{REFERENCES}

[1] Li, X.; Lopes, L. A. C.; Williamson,S. S. (2009). On the suitability of plug-in hybrid electric vehicle (PHEV) charging infrastructures based on wind and solar energy, Proceedings of the IEEE Power \& Energy Society General Meeting, 8 pages, doi:10.1109/PES.2009.5275171

[2] Dickerman, L.; Harrison, J. (2010). A new car, a new grid, IEEE Power and Energy Magazine, Vol. 8, No. 2, 55-61,doi:10.1109/MPE.2009.935553

[3] Webster, R. (1999). Can the electricity distribution network cope with an influx of electric vehicles?, Journal of Power Sources, Vol. 80, No. 1-2, 217-225, doi:10.1016/S03787753(98)00262-6

[4] Tamor, M. A.; Gearhart, C.; Soto, C. (2013). A statistical approach to estimating acceptance of electric vehicles and electrification of personal transportation, Transportation Research Part C: Emerging Technologies, Vol. 26, 125-134, doi:10.1016/j.trc.2012.07.007

[5] Nie, Y.; Ghamami, M. (2013). A corridor-centric approach to planning electric vehicle charging infrastructure, Transportation Research Part B: Methodological, Vol. 57, 172-190, doi:10.1016/ j.trb.2013.08.010

[6] Liu, S.; Gong, D. (2014). Modelling and simulation on recycling of electric vehicle batteries using agent approach, International Journal of Simulation Modelling, Vol. 13, No. 1, 79-92, doi:10.2507/IJSIMM13(1)CO1

[7] Yilmaz, M.; Krein, P. T. (2013). Review of battery charger topologies, charging power levels, and infrastructure for plug-in electric and hybrid vehicles, IEEE Transactions on Power Electronics, Vol. 28, No. 5, 2151-2169, doi:10.1109/TPEL.2012.2212917

[8] Hutson, C.; Venayagamoorthy, G. K.; Corzine, K. A. (2008). Intelligent scheduling of hybrid and electric vehicle storage capacity in a parking lot for profit maximization in grid power transactions, IEEE Energy 2030 Conference Proceedings, 8 pages, doi:10.1109/ENERGY.2008.4781051

[9] Dashora, Y.; Barnes, J. W.; Pillai, R. S.; Combs, T. E.; Hilliard, M.; Chinthavali, M. S. (2010). The PHEV charging infrastructure planning (PCIP) problem, International Journal of Emerging Electric Power Systems, Vol. 11, No. 2, doi:10.2202/1553-779X.2482

[10] Kuby, M.; Lim, S. (2005). The flow-refueling location problem for alternative-fuel vehicles, Socio-Economic Planning Sciences, Vol. 39, No. 2, 125-145, doi:10.1016/j.seps.2004.03.001

[11] Lim, S.; Kuby, M. (2010). Heuristic algorithms for siting alternative-fuel stations using the flow-refueling location model, European Journal of Operational Research, Vol. 204, No. 1, 51-61, doi:10.1016/j.ejor.2009.09.032

[12] Wang, Y.-W.; Lin, C.-C. (2013). Locating multiple types of recharging stations for battery-powered electric vehicle transport, Transportation Reserach Part E: Logistics Transportation Review, Vol. 58, 76-87, doi:10.1016/j.tre.2013.07.003

[13] Mak, H.-Y.; Rong, Y.; Shen, Z.-J. M. (2013). Infrastructure planning for electric vehicles with battery swapping, Management Science, Vol. 59, No. 7, 1557-1575, doi:10.1287/mnsc.1120.1672

[14] Axsen, J.; Kurani, K. S. (2010). Anticipating plug-in hybrid vehicle energy impacts in California: constructing consumer-informed recharge profiles, Transportation Research Part D: Transport and Environment, Vol. 15, No. 4, 212-219, doi:10.1016/j.trd.2010.02.004 
[15] Lu, X. C.; Chen, Q. B.; Zhang, Z. J. (2014). The electric vehicle routing optimizing algorithm and the charging stations' layout analysis in Beijing, International Journal of Simulation Modelling, Vol. 13, No. 1, 116-127, doi:10.2507/IJSIMM13(1)CO4

[16] Green II, R. C.; Wang, L.; Alam, M. (2010). The impact of plug-in hybrid electric vehicles on distribution networks: A review and outlook, Renewable and Sustainable Energy Reviews, Vol. 15, No. 1, 544-553, doi:10.1016/j.rser.2010.08.015

[17] Cavadas, J.; de Almeida Correia, G. H.; Gouveia, J. (2015). A MIP model for locating slow-charging stations for electric vehicles in urban areas accounting for driver tours, Transportation Research Part E: Logistics and Transportation Review, Vol. 75, 188-201, doi: $10.1016 /$ j.tre.2014.11.005

[18] Axsen, J.; Kurani, K. S. (2010). Anticipating plug-in hybrid vehicle energy impacts in California: Constructing consumer-informed recharge profiles, Transportation Research Part D: Transport and Environment, Vol. 15, No. 4, 212-219, doi:10.1016/j.trd.2010.02.004

[19] Raviv, T. (2012). The battery switching station scheduling problem, Operations Research Letters, Vol. 40, No. 6, 546-550, doi:10.1016/j.orl.2012.09.007

[20] He, F.; Wu, D.; Yin, Y.; Guan, Y. (2013). Optimal deployment of public charging stations for plug-in hybrid electric vehicles, Transportation Research Part B: Methodological, Vol. 47, 87-101, doi: $\underline{10.1016 / j . t r b .2012 .09 .007}$ 